\title{
Phase Transitions in the Water-Hydrogen System at Pressures up to 4.7 kbar
}

\author{
Vladimir E. Antonov, ${ }^{*, \dagger}$ Vadim S. Efimchenko, ${ }^{\dagger}$ and Marek Tkacz \\ Institute of Solid State Physics, Russian Academy of Sciences, 142432 Chernogolovka, Moscow District, Russia, \\ and Institute of Physical Chemistry, Polish Academy of Sciences, Kasprzaka 44/52, 01-224 Warsaw, Poland
}

Received: September 9, 2008; Revised Manuscript Received: November 14, 2008

Using a volumetric technique, a $T-P$ diagram of phase transformations between the hydrogen-rich clathrate hydrate (sII phase), hydrogen-filled ice II ( $\mathrm{C}_{1}$ phase), and the liquid (L) is studied in the $\mathrm{H}_{2} \mathrm{O}-\mathrm{H}_{2}$ system at pressures up to $4.7 \mathrm{kbar}$ and temperatures from -22 to $+15{ }^{\circ} \mathrm{C}$. The volume and entropy effects of these transformations are established in the vicinity of the triple point of the $\mathrm{L}+\mathrm{sII}+\mathrm{C}_{1}$ equilibrium located at $P=3.6(1)$ kbar and $T=+1(1){ }^{\circ} \mathrm{C}$. The estimated molar ratios $\mathrm{H}_{2} / \mathrm{H}_{2} \mathrm{O}$ of phases at the triple point are $X_{\mathrm{L}}$ $=0.04(2), X_{\mathrm{SII}}=0.32(2)$, and $X_{\mathrm{C} 1}=0.10(2)$.

\section{Introduction}

Figure 1 presents the available experimental data on phase relations in water ${ }^{1}$ (dashed lines) and in water placed in an atmosphere of molecular hydrogen (solid lines) at pressures up to 7 kbar. The melting line of ices in a $\mathrm{H}_{2}$ atmosphere was constructed by differential thermal analysis ${ }^{2}$ (thin solid line in Figure 1) at pressures up to $15 \mathrm{kbar}$ and by optical microscopy ${ }^{3}$ at pressures 7-32 kbar. The experiments showed that the melting temperature of low-pressure hexagonal ice $I_{h}$ in hydrogen nearly coincides with that in the absence of hydrogen at pressures up to $1 \mathrm{kbar}$. At about 1, 3.6, and also $25.5 \mathrm{kbar}$ (not shown in Figure 1), the melting line of ices makes sharp bends signaling the formation of new solid phases rich in hydrogen.

The three hydrogen-rich $\mathrm{H}_{2} \mathrm{O}-\mathrm{H}_{2}$ phases are called hydrogen clathrate hydrates, and their crystal structures are networks of hydrogen-bonded $\mathrm{H}_{2} \mathrm{O}$ molecules encasing guest $\mathrm{H}_{2}$ molecules that only weakly interact with the network by van der Waals forces. Neutron diffraction investigations showed that the hydrate formed in the pressure interval $1-3.6 \mathrm{kbar}$ has an sIItype crystal structure. ${ }^{4}$ Its cubic unit cell is composed of 136 $\mathrm{H}_{2} \mathrm{O}$ molecules and, depending on the pressure and temperature, can accommodate from $27.2^{5}$ to $48^{6}$ guest molecules of $\mathrm{H}_{2}$, which gives a molar ratio $\mathrm{H}_{2} / \mathrm{H}_{2} \mathrm{O}$ of $X=0.200-0.353$.

The hydrates stable at higher hydrogen pressures were denoted $\mathrm{C}_{1}$ and $\mathrm{C}_{2}$ and studied by Raman spectroscopy and $\mathrm{X}$-ray diffraction. ${ }^{3}$ The $\mathrm{C}_{1}$ phase, which is expected to be stable at hydrogen pressures $3.6-25.5 \mathrm{kbar}$, was examined by X-rays at $21 \mathrm{kbar}$ and $22{ }^{\circ} \mathrm{C}$. The structure of its $\mathrm{H}_{2} \mathrm{O}$ sublattice was shown to be similar to that of rhombohedral high-pressure ice II (the stability field of this ice is labeled "II" in Figure 1). On the basis of results of the Raman studies, the hydrogen content of the $\mathrm{C}_{1}$ phase was assumed to be invariable within the examined $T-P$ range and equal to $X=1 / 6$, with the $\mathrm{H}_{2}$ molecules occupying each of the six accessible cages in the unit cell composed of $36 \mathrm{H}_{2} \mathrm{O}$ molecules. Similar investigations demonstrated $^{3}$ that the $\mathrm{C}_{2}$ phase had a diamond-like $\mathrm{H}_{2} \mathrm{O}$ sublattice and $X=1$ at a hydrogen pressure of $31 \mathrm{kbar}$ and room temperature.

\footnotetext{
* To whom correspondence should be addressed. E-mail: antonov@ issp.ac.ru.

${ }^{\dagger}$ Russian Academy of Sciences.

¥ Polish Academy of Sciences.
}

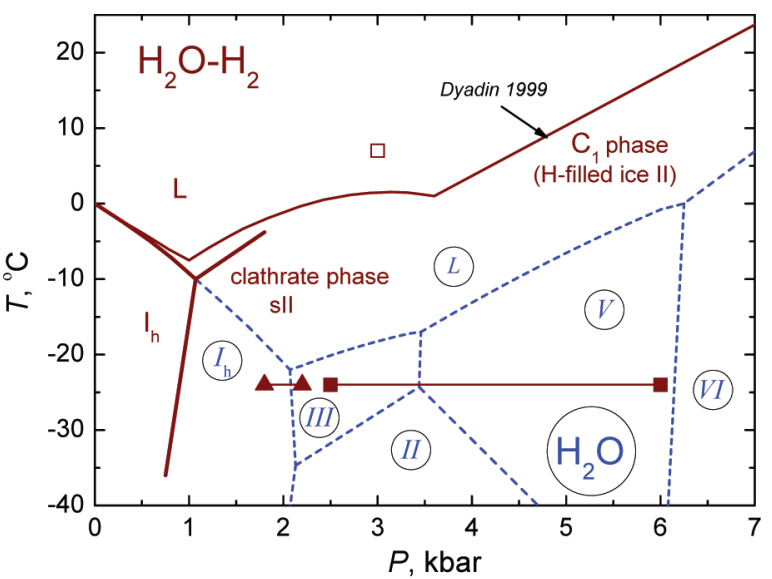

Figure 1. $T-P$ diagram of phase transitions in the $\mathrm{H}_{2} \mathrm{O}-\mathrm{H}_{2}$ system with the $\mathrm{H}_{2}$ gas taken in access (solid lines) superimposed onto the equilibrium diagram of $\mathrm{H}_{2} \mathrm{O}^{1}$ (dashed lines; phase fields are labeled with the encircled symbols). The upper thin solid curve shows the melting line of water ices in a hydrogen atmosphere determined by differential thermal analysis. ${ }^{2}$ Three thick solid lines radiating from the $\mathrm{L}+\mathrm{I}_{\mathrm{h}}+$ sII triple point are constructed using a volumetric technique and represent phase equilibria between the $\mathrm{L}, \mathrm{I}_{\mathrm{h}}$, and sII phases. The solid triangles ${ }^{6}$ and solid squares ${ }^{9}$ connected with the horizontal lines indicate the $\mathrm{L} \rightarrow$ sII transitions, and the open square ${ }^{9}$ stands for the sII $\rightarrow \mathrm{L}$ transition, all determined by optical microscopy in diamond anvils.

Phase relations in the $\mathrm{H}_{2} \mathrm{O}-\mathrm{H}_{2}$ system are of significant interest, in the first place, for planetary science because hydrogen and water are among the basic building materials of many planets. In this connection, studies of the sII hydrogen hydrate are most attractive as its synthesis pressure is within the range of interior conditions of small, icy satellites, and transformations of the sII phase to other phases may play a key role in the evolution of these icy bodies. ${ }^{4}$

Using a volumetric technique, we have earlier examined transitions between the sII and $\mathrm{I}_{\mathrm{h}}$ and $\mathrm{L}$ (liquid) phases in the $\mathrm{H}_{2} \mathrm{O}-\mathrm{H}_{2}$ system ${ }^{7,5}$ and $\mathrm{D}_{2} \mathrm{O}-\mathrm{D}_{2}$ system $^{5,8}$ at pressures up to $1.8 \mathrm{kbar}$. The $T-P-X$ diagrams of these systems proved to be very similar, and their comparative analysis allowed us to establish rather accurately the position of the $\mathrm{I}_{\mathrm{h}} \leftrightarrow$ sII equilibrium line, the $X(P)$ dependence for the sII phase, and the hydrogen contents of the sII and $\mathrm{I}_{\mathrm{h}}$ and $\mathrm{L}$ phases at the triple point of the $\mathrm{L}+\mathrm{I}_{\mathrm{h}}+$ sII equilibrium. ${ }^{8}$ The constructed portion 
of the equilibrium $T-P$ diagram of the $\mathrm{H}_{2} \mathrm{O}-\mathrm{H}_{2}$ system is shown in Figure 1 by the thick solid lines.

In the present work, using another type of volumetric technique, we constructed the line of the sII $\leftrightarrow \mathrm{C}_{1}$ transformation, which is the high-pressure boundary of the stability region of the sII phase, and estimated the hydrogen contents of the sII and $\mathrm{C}_{1}$ and $\mathrm{L}$ phases near the second triple point of the $\mathrm{L}+$ sII $+\mathrm{C}_{1}$ equilibrium. We also re-examined the position of this triple point because experimental results of ref 9 (open and solid squares in Figure 1) did not agree well with the melting curve of ices in the $\mathrm{H}_{2} \mathrm{O}-\mathrm{H}_{2}$ system constructed in ref 2 (thin solid curve in Figure 1).

\section{Experimental Details}

The experiments were carried out in a piston-cylinder highpressure chamber ${ }^{10}$ with an inner diameter of $12 \mathrm{~mm}$, in which gaseous hydrogen was compressed or decompressed by a movement of the piston. The movement was followed by a precise mechanical indicator providing the accuracy $\pm 1 \mathrm{~mm}^{3}$ in the determination of the volume change. The pressure was measured by a manganin wire gauge accurate to within \pm 30 bar. The temperature was regulated with an accuracy of \pm 0.5 ${ }^{\circ} \mathrm{C}$ by circulating the cooling liquid through the jacket of the high-pressure chamber.

Each studied sample of $\mathrm{H}_{2} \mathrm{O}$ was frozen and powdered in an agate mortar under liquid nitrogen prior to the high-pressure experiment in order to speed up the kinetics of hydrogen absorption and desorption. The powder was poured into the high-pressure chamber cooled below $-23^{\circ} \mathrm{C}$; the chamber was filled with gaseous hydrogen under a pressure of $1.5 \mathrm{kbar}$, and the gas was further compressed to $2.2-2.5 \mathrm{kbar}$. The sample was exposed to these conditions until its transformation to the sII phase was complete, and the volume or the temperature in the chamber was then varied as required. The preliminary cooling to $-23{ }^{\circ} \mathrm{C}$ was necessary to avoid the melting of the starting sample of frozen water, which had no time to absorb enough hydrogen and to transform to the sII phase, on crossing the melting line of ice $\mathrm{I}_{\mathrm{h}}$ (see Figure 1).

The mass of the $\mathrm{H}_{2} \mathrm{O}$ samples was limited to $1.3-1.7 \mathrm{~g}$ so as not to damage the electrical leads inside the chamber by the densified ice powder at the maximum compression. The total mass of hydrogen in the high-pressure chamber was $0.4-0.45$ g. This gave a molar ratio of $\mathrm{H}_{2} / \mathrm{H}_{2} \mathrm{O}>2$; therefore, the hydrogen gas in the chamber was always in excess during our experiments.

The solubility of water in gaseous hydrogen was earlier shown to be vanishingly small at $27^{\circ} \mathrm{C}$ and pressures up to 6 kbar. ${ }^{9}$ We therefore assumed that the gaseous phase in our highpressure experiments was pure hydrogen and used the equation of state of $\mathrm{H}_{2}$ from ref 11 to describe its $P-V-T$ relations.

\section{Results and Discussion}

$\boldsymbol{T}-\boldsymbol{P}$ Phase Diagram. The obtained diagram of phase transformations in the $\mathrm{H}_{2} \mathrm{O}-\mathrm{H}_{2}$ system is shown in Figure 2.

The sII $\leftrightarrow \mathrm{C}_{1}$ transformation was examined by constructing isotherms of pressure, $P$, measured in the course of a stepwise increase and decrease in the total volume, $V$, of the $\mathrm{H}_{2} \mathrm{O}-\mathrm{H}_{2}$ system. The volume itself could not be accurately measured and was counted from a value arbitrary chosen in each experiment. Changing the volume in the high-pressure chamber led to a temporal drift of the pressure. The drift lasted less than $5 \mathrm{~min}$ in the absence of phase transitions and about $20 \mathrm{~min}$ in the intervals of the sII $\rightarrow \mathrm{C}_{1}$ and $\mathrm{C}_{1} \rightarrow$ sII transitions. While constructing isotherms, the volume of the system was held constant until the pressure stopped changing and that final value

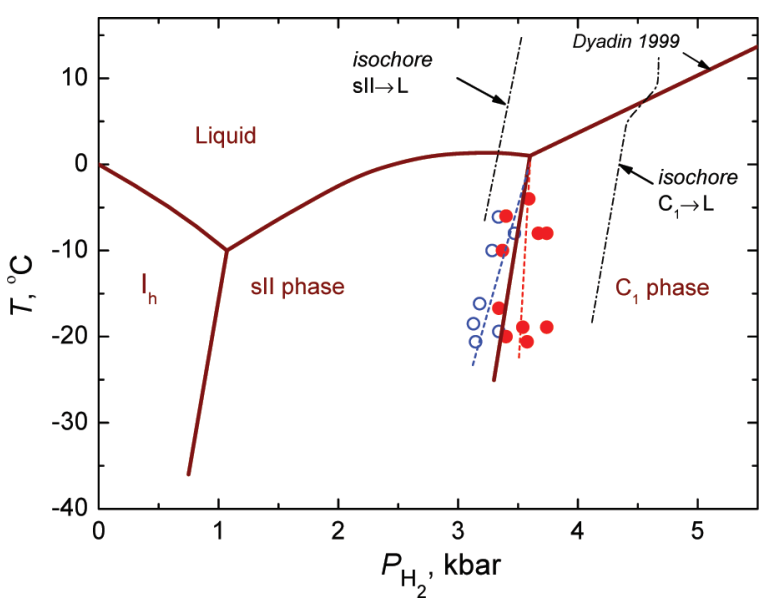

Figure 2. $T-P$ phase diagram of the $\mathrm{H}_{2} \mathrm{O}-\mathrm{H}_{2}$ system according to results of the present paper. The thick solid lines show equilibria between the corresponding phases. The lines of equilibria between pairs of the $\mathrm{L}, \mathrm{I}_{\mathrm{h}}$, and sII phases at pressures below $1.8 \mathrm{kbar}$ are taken from ref 8. The line of the $\mathrm{C}_{1} \leftrightarrow \mathrm{L}$ equilibrium at pressures above $3.6 \mathrm{kbar}$ is from ref 2. The line of the sII $\leftrightarrow \mathrm{C}_{1}$ equilibrium is plotted in the middle between the solid and open circles representing respectively the points of the sII $\rightarrow \mathrm{C}_{1}$ and $\mathrm{C}_{1} \rightarrow$ sII transition. The thin dash-dot lines are the isochors from Figure 4.

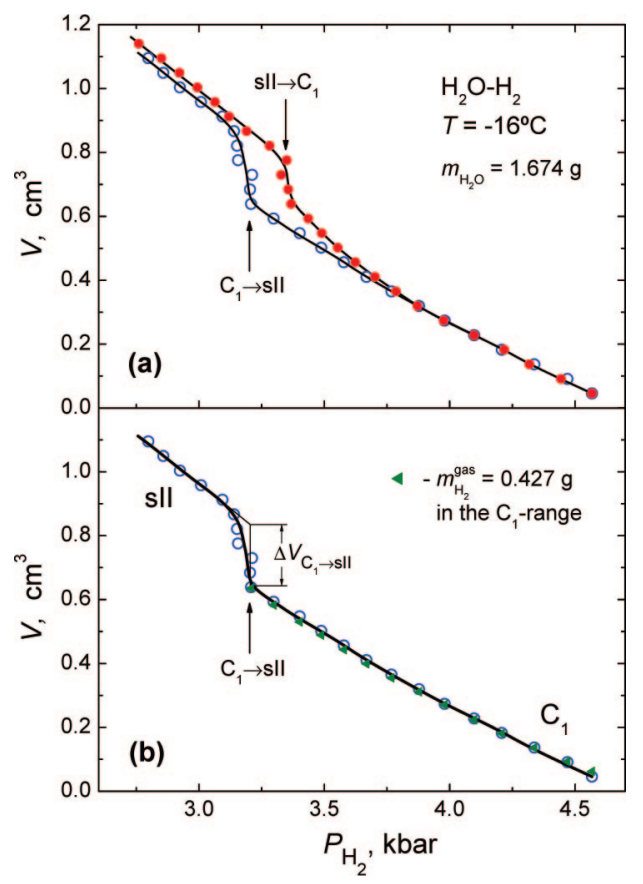

Figure 3. Variation of volume of the $\mathrm{H}_{2} \mathrm{O}-\mathrm{H}_{2}$ system as a function of hydrogen pressure at a temperature of $-16{ }^{\circ} \mathrm{C}$. The solid and open circles refer to increasing and decreasing pressure, respectively. The vertical arrows in (a) show the points of the sII $\rightarrow \mathrm{C}_{1}$ and $\mathrm{C}_{1} \rightarrow$ sII transitions indicated in Figure 2. The solid left triangles in (b) show the results of fitting the experimental points (open circles) for the $\mathrm{C}_{1}$ phase (see subsection "Hydrogen Content...").

was plotted in the figure. Representative isotherms $P(V)$ are shown in Figure 3a in a more convenient form, with the $P$-axis directed horizontally. The points of the sII $\rightarrow \mathrm{C}_{1}$ and $\mathrm{C}_{1} \rightarrow$ sII transitions indicated in Figure 2 were determined from an abrupt increase in the duration of the pressure drift arising after the increase and decrease in volume, respectively.

The temperature dependences of pressures of the sII $\rightarrow \mathrm{C}_{1}$ and $\mathrm{C}_{1} \rightarrow$ sII transitions in Figure 2 are approximated with thin dashed lines, and the thick solid line representing the sII $\leftrightarrow \mathrm{C}_{1}$ equilibrium is plotted in the middle between them. The line of 


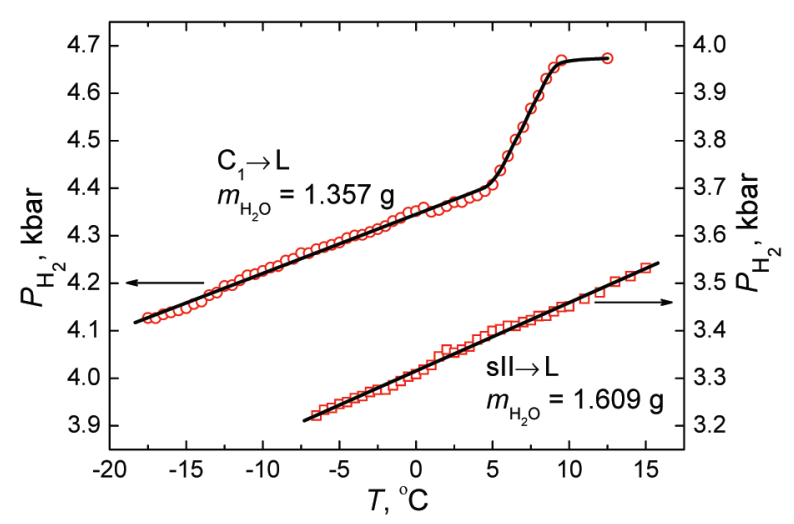

Figure 4. Variation of pressure in the $\mathrm{H}_{2} \mathrm{O}-\mathrm{H}_{2}$ system at constant volume measured in the regime of heating at a rate of $0.25^{\circ} \mathrm{C} / \mathrm{min}$.

the sII $\leftrightarrow \mathrm{C}_{1}$ equilibrium thus constructed crosses the melting line of ices determined in ref 2 (thin solid curve in Figure 1) exactly at the point $3.6 \mathrm{kbar}$ and $1{ }^{\circ} \mathrm{C}$, where the curve makes a sharp bend.

This bending point was assumed ${ }^{2}$ to well reproduce the point of invariant equilibrium of the liquid with the sII and $\mathrm{C}_{1}$ phases because the melting temperature of most substances is very close to the temperature of phase equilibrium between the solid and liquid. The observed intersection of the three independently determined phase boundaries at one point strongly corroborates this assumption.

We also constructed two isochors intersecting the melting curves of the sII and $\mathrm{C}_{1}$ phases near the $\mathrm{L}+\mathrm{sII}+\mathrm{C}_{1}$ triple point. The isochors are shown in Figure 4. The smooth curves fitting these isochors are copied to Figure 2 as dash-dot lines.

On the upper isochor in Figure 4, there is a step at temperatures $5-9{ }^{\circ} \mathrm{C}$. The position of this step well agrees with the melting curve of the $\mathrm{C}_{1}$ phase constructed in ref 2 and drawn with a thick solid line in Figure 2. The good agreement between the melting conditions determined by different techniques suggests that the melting curve from ref 2 should be accurate as a whole, and it should well represent the $\mathrm{C}_{1} \leftrightarrow$ L equilibrium. Furthermore, the melting curve of the sII phase measured in ref 2 should accurately reproduce the sII $\leftrightarrow \mathrm{L}$ equilibrium near the $\mathrm{L}+\mathrm{sII}+\mathrm{C}_{1}$ triple point; otherwise, it would not meet with the other two equilibrium lines at this point.

At the same time, it was mentioned in ref 2 that melting of the sII phase was a very slow process, and the equilibrium was not achieved in some DTA experiments. In fact, we earlier observed $^{5}$ a certain overheating of the sII phase in the DTA measurements at pressures below $1.8 \mathrm{kbar}$ (compare the melting curves plotted by the thin and thick solid lines in Figure 1). To check whether the DTA curve well represents the sII $\leftrightarrow \mathrm{L}$ equilibrium in the range of the shallow maximum located at 3.0-3.4 kbar, we constructed an isochor crossing this melting curve, $T_{\mathrm{m}}(P)$, at a pressure of $3.3 \mathrm{kbar}$. The isochor is shown at the bottom of Figure 4. As one can see, it is close to a straight line and shows no stepwise anomalies. This implies a zero volume effect, $\Delta V_{\mathrm{m}}$, of melting and therefore justifies the occurrence of a maximum on the melting curve. (Clapeyron's equation with $\Delta V_{\mathrm{m}}=0$ gives $\mathrm{d} T_{\mathrm{m}} / \mathrm{d} P=\Delta V_{\mathrm{m}} / \Delta S_{\mathrm{m}}=0$ because the change in entropy, $\Delta S_{\mathrm{m}}$, is always positive on melting.) Correspondingly, we extended the equilibrium melting curve from ref 5 up to the $\mathrm{L}+\mathrm{sII}+\mathrm{C}_{1}$ triple point (thick solid line in Figure 2) so that it asymptotically approached the melting line from ref 2 near its maximum.

The melting point of the sII phase from ref 9 (open square in Figure 1) is located rather far above the melting line thus constructed and should therefore be considered as a nonequilibrium result. As for the reverse transition-crystallization of the sII phase from the liquid at decreasing temperature-it always requires significant supercooling. $., 9,7$

At pressures up to $1.8 \mathrm{kbar}$, the sII phase crystallizes together with ice $\mathrm{I}_{\mathrm{h}}$, some $4{ }^{\circ} \mathrm{C}$ below the melting curve of this ice shown in Figure 1 by the dashed line. ${ }^{7}$ The effect of simultaneous crystallization of the sII phase and metastable ice $I_{h}$ was explained in ref 8. Namely, the growing particles of ice $\mathrm{I}_{\mathrm{h}}$ should supersaturate the surrounding of water with hydrogen, thus providing more favorable conditions for the nucleation and growth of the hydrogen-rich sII phase. In its turn, the growing sII phase depletes the water of the dissolved hydrogen, therefore facilitating the nucleation and growth of the hydrogen-poor ice $I_{h}$. The formation of the sII phase on cooling the liquid to -24 ${ }^{\circ} \mathrm{C}$ at pressures near 2 kbar observed in ref 6 (solid triangles in Figure 1) agrees with results of refs 7 and 8 . The formation of the sII phase at the same temperature and pressures up to 6 $\operatorname{kbar}^{9}$ (solid squares in Figure 1), deep inside the stability region of the $C_{1}$ phase, needs further investigation.

Volume and Entropy Effects of Phase Transitions near the $\mathbf{L}+\mathbf{s I I}+\mathbf{C}_{\mathbf{1}}$ Triple Point. The volume effect of the sII $\rightarrow \mathrm{C}_{1}$ transition was determined from the experimental isotherms $\Delta V(P)$.

As seen from Figure 3a, the isotherms measured at increasing and decreasing pressure do not coincide in the interval about 0.5 kbar above the sII $\rightarrow \mathrm{C}_{1}$ transition. The observed difference apparently reflects the incompleteness of this transition at increasing pressure. Below the reverse $\mathrm{C}_{1} \rightarrow$ sII transition, the isotherms do not coincide either; only in this case they go parallel to each other and do not converge. Consequently, the difference between the isotherms in this pressure range should rather be attributed to a certain systematic error accumulated during the whole cycle of the pressure increase and decrease (e.g., caused by a slight gas escape from the high-pressure chamber). We therefore assumed that the $\mathrm{C}_{1} \rightarrow$ sII transition was nearly complete right below the interval of the steep volume increase. The volume effect of the transition was determined as shown in Figure 3b. Within the experimental error, the magnitude of this effect calculated per $1 \mathrm{~g}$-mol of $\mathrm{H}_{2} \mathrm{O}$ did not depend on the temperature in the examined interval from -21 to $-4{ }^{\circ} \mathrm{C}$. Taking into account that the linear fit to the experimental points of the $\mathrm{C}_{1} \rightarrow$ sII transition (dashed line in Figure 2) passed through the $\mathrm{L}+\mathrm{sII}+\mathrm{C}_{1}$ triple point at a nearby temperature of $+1{ }^{\circ} \mathrm{C}$, the mean value of $\Delta V_{\mathrm{sII} \rightarrow \mathrm{C}_{1}}=$ $2.01(3) \mathrm{cm}^{3} / \mathrm{mol}$ was ascribed to the $\mathrm{C}_{1} \rightarrow$ sII transition near the triple point.

The volume effects of the $\mathrm{C}_{1} \rightarrow \mathrm{L}$ and $\mathrm{L} \rightarrow$ sII transitions could not be determined from experiment, and we calculated them using the additive relations for the three changes in volume, $\Delta V_{i}$, and three changes in entropy, $\Delta S_{i}$, accompanying phase transitions around the $\mathrm{L}+\mathrm{sII}+\mathrm{C}_{1}$ triple point:

$$
\sum \Delta V_{i}=0 \text { and } \sum \Delta S_{i}=\sum(\mathrm{d} T / \mathrm{d} P)_{i} / \Delta V_{i}=0
$$

Using experimental values of $\Delta V_{\mathrm{sII} \rightarrow \mathrm{C}_{1}}$ and the slopes $(\mathrm{d} T /$ $\mathrm{d} P)_{i}$ of the three lines of phase transitions radiating from the triple point, these two equations give the two missing changes in volume, $\Delta V_{\mathrm{sII} \rightarrow \mathrm{C}_{1}}$ and $\Delta V_{\mathrm{sII} \rightarrow \mathrm{C}_{1}}$. The obtained values of $\Delta V_{i}$, $(\mathrm{d} T / \mathrm{d} P)_{i}, \Delta S_{i}=(\mathrm{d} T / \mathrm{d} P)_{i} / \Delta V_{i}$, and changes in enthalpy $\Delta H_{i}=$ $T \Delta S_{i}$ near the $\mathrm{L}+\mathrm{sII}+\mathrm{C}_{1}$ triple point are listed in Table 1. For comparison, Table 1 also presents analogous data for phase transitions near the $\mathrm{L}+\mathrm{I}_{\mathrm{h}}+$ sII triple point taken from ref 8 .

An analysis of the available experimental data made it possible to produce estimates of the molar volumes and 
TABLE 1: Parameters of Phase Transformations in the $\mathrm{H}_{2} \mathrm{O}-\mathrm{H}_{2}$ System near the Triple Points of the $\mathrm{L}+\mathrm{sII}+\mathrm{C}_{1}$ Equilibrium (Data of the Present Work) and $\mathbf{L}+\mathbf{I}_{\mathbf{h}}+$ sII Equilibrium (Ref 8) ${ }^{a}$

\begin{tabular}{clrccr}
\hline & & $\Delta V$, & $\mathrm{d} T / \mathrm{d} P$, & $\Delta S$, & \multicolumn{1}{c}{$\Delta H}$, \\
triple point & transition & $\mathrm{cm}^{3} / \mathrm{mol}$ & $\mathrm{K} / \mathrm{kbar}$ & $\mathrm{J} /(\mathrm{K}$ mol $)$ & $\mathrm{kJ} / \mathrm{mol}$ \\
\hline $\mathrm{L}+\mathrm{sII}+\mathrm{C}_{1}$ & $\mathrm{sII} \rightarrow \mathrm{C}_{1}$ & -2.01 & 89 & -2.3 & -0.62 \\
$(3.6 \mathrm{kbar}, 294 \mathrm{~K})$ & $\mathrm{C}_{1} \rightarrow \mathrm{L}$ & 1.66 & 6.5 & 25.6 & 7.01 \\
& $\mathrm{~L} \rightarrow \mathrm{sII}$ & 0.35 & -1.5 & -23.3 & -6.39 \\
$\mathrm{~L}+\mathrm{I}_{\mathrm{h}}+\mathrm{sII}$ & $\mathrm{I}_{\mathrm{h}} \rightarrow \mathrm{sII}$ & -4.33 & 128 & -3.38 & -0.89 \\
$(1.07 \mathrm{kbar}, 263 \mathrm{~K})$ & $\mathrm{sII} \rightarrow \mathrm{L}$ & 2.05 & 8.5 & 24.1 & 6.34 \\
& $\mathrm{~L} \rightarrow \mathrm{I}_{\mathrm{h}}$ & 2.28 & -11.0 & -20.7 & -5.44
\end{tabular}

${ }^{a}$ The changes in volume $(\Delta V)$, entropy $(\Delta S)$, and enthalpy $(\Delta H)$ are calculated per $1 \mathrm{~g}$-mol of $\mathrm{H}_{2} \mathrm{O}$.

TABLE 2: Molar Ratio $\left(X=\mathrm{H}_{2} / \mathrm{H}_{2} \mathrm{O}\right)$ and Molar Volume $(V)$ of Phases in the $\mathrm{H}_{2} \mathrm{O}-\mathrm{H}_{2}$ System at the Triple Points $\mathrm{L}$ + sII $+\mathbf{C}_{1}$ (Estimates of the Present Work) and $\mathbf{L}+\mathbf{I}_{\mathbf{h}}+$ sII (Results of Ref 8)

\begin{tabular}{lccccc}
\hline & \multicolumn{2}{c}{$\mathrm{L}+\mathrm{sII}+\mathrm{C}_{1}$ triple point } & & $\mathrm{L}+\mathrm{I}_{\mathrm{h}}+$ sII triple point \\
\cline { 2 - 3 } phase & $X$ & $V, \mathrm{~cm}^{3} / \mathrm{mol}$ & & $X$ & $V, \mathrm{~cm}^{3} / \mathrm{mol}$ \\
\hline $\mathrm{L}$ & $0.04(2)$ & 16.31 & & $0.017(5)$ & 17.31 \\
sII & $0.32(2)$ & 21.77 & & $0.207(5)$ & 22.16 \\
$\mathrm{C}_{1}$ & $0.10(2)$ & $(15.21)$ & & \\
$\mathrm{I}_{\mathrm{h}}$ & & & & $0.011(5)$ & 19.36 \\
$\mathrm{H}_{2}$ gas & & 20.73 & & & 36.41
\end{tabular}

equilibrium hydrogen contents of the $\mathrm{L}, \mathrm{sII}$, and $\mathrm{C}_{1}$ phases near the $\mathrm{L}+\mathrm{sII}+\mathrm{C}_{1}$ triple point. The results are presented in Table 2 together with our earlier data ${ }^{8}$ for phases near the $\mathrm{L}+\mathrm{I}_{\mathrm{h}}+$ sII point. Two follow-up sections of this paper describe how the new results were obtained.

Molar Volumes of the $\mathbf{L}$, sII, and $\mathbf{C}_{1}$ Phases. The dependence $V_{\mathrm{L} 0}(T, P)$ of the molar volume of liquid $\mathrm{H}_{2} \mathrm{O}$ without hydrogen in a wide $P-T$ range was accurately measured many years ago. ${ }^{12}$ At infinite dilution in water, hydrogen increases $V_{\mathrm{L} 0}$ by a value of $\partial V_{\mathrm{L}} / \partial X_{\mathrm{L}}=7.2^{13}$ or $8.7^{14} \mathrm{~cm}^{3} / \mathrm{mol} \mathrm{H}_{2}$. We adopted the average $\beta=8.0 \mathrm{~cm}^{3} / \mathrm{mol} \mathrm{H}_{2}$ of these two $\partial V_{\mathrm{L}} / \partial X_{\mathrm{L}}$ values and calculated the molar volume of water under high hydrogen pressure as

$$
V_{\mathrm{L}}=V_{\mathrm{L} 0}+\beta X_{\mathrm{L}}
$$

using the experimental $V_{\mathrm{L} 0}(T, P)$ values from ref 12 .

The sII phase in the $\mathrm{H}_{2} \mathrm{O}-\mathrm{H}_{2}{ }^{4}$ and $\mathrm{D}_{2} \mathrm{O}-\mathrm{D}_{2}{ }^{4-6}$ systems was earlier investigated by $\mathrm{X}$-ray and neutron diffraction at pressures up to $2.2 \mathrm{kbar}$ and temperatures below $-39^{\circ} \mathrm{C}$. An analysis of the obtained results showed that at temperatures of interest, from -21 to $+1{ }^{\circ} \mathrm{C}$, the molar volume of the sII phase in the $\mathrm{H}_{2} \mathrm{O}-\mathrm{H}_{2}$ system can be approximated by the linear equation

$$
V_{\text {sII }}=22.37-0.167 P
$$

where $P$ is in kbar and $V_{\text {sII }}$ is in units of $\mathrm{cm}^{3} / \mathrm{mol} \mathrm{H}_{2} \mathrm{O}$. We used this equation to estimate $V_{\text {sII }}$ at hydrogen pressures up to $3.6 \mathrm{kbar}$, including the value of $V_{\text {sII }}$ at the $\mathrm{L}+\mathrm{sII}+\mathrm{C}_{1}$ triple point indicated in Table 2.

As for the $C_{1}$ phase, it was earlier synthesized only once, ${ }^{3}$ and the solid square in Figure 5 shows the only known value of its molar volume, $V_{\mathrm{C}_{1}}$, determined by $\mathrm{X}$-ray diffraction at 21 kbar and $22{ }^{\circ} \mathrm{C}$. As one can see, this point is far from the pressure region 3-4.7 kbar, where the $\mathrm{C}_{1}$ phase was investigated in the present work. Nevertheless, a rather plausible estimation

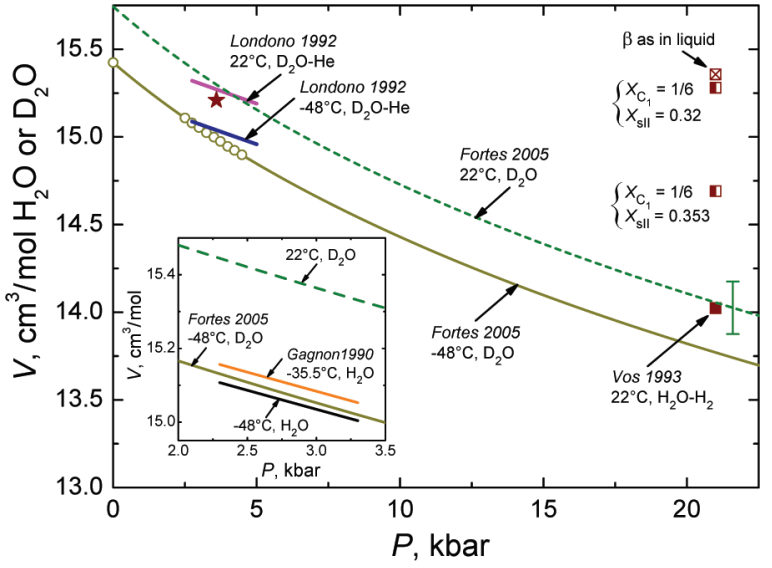

Figure 5. Pressure dependences of the molar volumes of gas-free ice II and of ice II filled with helium and hydrogen (the $C_{1}$ phase). The solid square stands for the $\mathrm{C}_{1}$ phase of hydrogen hydrate at $22{ }^{\circ} \mathrm{C} .{ }^{3}$ The circles and the solid line drawn through them show the experimental results for heavy ice II at $-48{ }^{\circ} \mathrm{C}$ fitted with a Birch-Murnaghan equation..$^{15}$ The dashed curve is calculated for ice II at $22^{\circ} \mathrm{C}$ using the parameters of the equation of state determined in ref 15 (see text). The two solid segments labeled "Londono 1992" are for He-filled heavy ice II. $^{17}$ The solid star indicates our estimate of the volume of the hydrogen hydrate $\mathrm{C}_{1}$ at the $\mathrm{L}+\mathrm{sII}+\mathrm{C}_{1}$ triple point. The crossed square is discussed in subsection "Molar Volumes..."; the half-blackened squares in subsection "Hydrogen Content...". The inset compares the data for $\mathrm{D}_{2} \mathrm{O}^{15}$ and $\mathrm{H}_{2} \mathrm{O}^{16}$ ice II.

of $V_{\mathrm{C}_{1}}$ in this pressure region could be achieved via an analysis of the $P-V-T$ relations of the "parent" phase of ice $\mathrm{II}^{15,16}$ and helium-filled ice II $^{17,18}$ studied in more detail.

The solid line in Figure 5 is a third-order Birch-Murnaghan fit to the experimental isotherm (open circles) of the molar volume of heavy ice II at $-48{ }^{\circ} \mathrm{C}$ determined by neutron diffraction. ${ }^{15}$ The dashed line represents the isotherm at $22{ }^{\circ} \mathrm{C}$ calculated using a Birch-Murnaghan equation with the same bulk modulus $K_{0}=109 \mathrm{kbar}$ and its first derivative $K_{0}^{\prime}=10$ as at $-48{ }^{\circ} \mathrm{C}$, while the zero-pressure volume was increased from $V_{0}\left(-48{ }^{\circ} \mathrm{C}\right)=15.42 \mathrm{~cm}^{3} / \mathrm{mol}$ to $V_{0}\left(22{ }^{\circ} \mathrm{C}\right)=15.74 \mathrm{~cm}^{3} /$ $\mathrm{mol}$, in accordance with the polynomial fit ${ }^{15}$ to the room-pressure experimental data. As one can see from Figure 5, the experimental point for the $\mathrm{C}_{1}$ hydrogen hydrate (solid square) is close to the dashed line. If the $\mathrm{C}_{1}$ hydrate is considered as a hydrogenfilled ice II, this suggests a small volume effect of hydrogen uptake by ice II.

The magnitude and sign of this effect cannot be assessed because of the large uncertainty in the extrapolation of the molar volume of ice II to $21 \mathrm{kbar}$ and $22^{\circ} \mathrm{C}$ (see the vertical error bar crossing the dashed line). What can definitely be said is that the effect is small compared to the volume expansion caused by the hydrogen dissolution in liquid water. In fact, if the hydrogen hydrate had $X_{\mathrm{C}_{1}}=1 / 6$ as suggested in ref 3 and $\beta \approx$ $8 \mathrm{~cm}^{3} / \mathrm{mol} \mathrm{H}$ as water does, its molar volume would exceed that of ice II by a value of $\beta X_{\mathrm{C}_{1}} \approx 1.3 \mathrm{~cm}^{3} / \mathrm{mol}$. The maximum possible difference in the volumes of the $\mathrm{C}_{1}$ phase and ice II at $21 \mathrm{kbar}$ and $22{ }^{\circ} \mathrm{C}$ is smaller by an order of magnitude. To illustrate this, the crossed square in Figure 5 shows the molar volume of the $\mathrm{C}_{1}$ phase that it would have in the case of $\beta=$ $8 \mathrm{~cm}^{3} / \mathrm{mol} \mathrm{H}_{2}$.

Cavities in the crystal structure of ice II can also be filled with He atoms. ${ }^{17}$ Figure 5 presents two isotherms of the molar volume of such a hydrate studied by neutron diffraction. ${ }^{18}$ The isotherms are not very accurate because they were obtained as a linear fit to a few experimental points randomly distributed in the range of helium pressures $2.75-5 \mathrm{kbar}$ and temperatures 
from -78 to $-12{ }^{\circ} \mathrm{C}$. In particular, the difference in the compressibilities of "pure" and He-filled ice II is below the error level. Nevertheless, a comparison of the corresponding isotherms for the helium hydrate ${ }^{18}$ and ice II $^{15}$ clearly shows a nearly zero volume expansion of ice II with increasing helium content, which reached $\mathrm{He} / \mathrm{D}_{2} \mathrm{O} \approx 1 / 12$ to $1 / 6$ depending on the temperature and helium pressure. ${ }^{18}$ This result can be considered as evidence in favor of the assumption discussed above that the molar volume of ice II nearly does not change when the analogues hydrogen hydrate is formed.

The inset to Figure 5 depicts isotherms of the molar volume of $\mathrm{H}_{2} \mathrm{O}$ ice II measured by piezometry at $-35.5{ }^{\circ} \mathrm{C}^{16}$ and recalculated for $-48{ }^{\circ} \mathrm{C}$ using the expansivity of $\mathrm{D}_{2} \mathrm{O}$ ice II. ${ }^{15}$ The near position of the isotherms for $\mathrm{H}_{2} \mathrm{O}$ and $\mathrm{D}_{2} \mathrm{O}$ ice II at $-48{ }^{\circ} \mathrm{C}$ shows that the isotopic variation of the molar volume of ice II is negligibly small compared with the uncertainty in the volume effect of hydrogen uptake.

On the grounds of the said, we assumed that the $\mathrm{C}_{1}$ phase in the $\mathrm{H}_{2} \mathrm{O}-\mathrm{H}_{2}$ system has the same molar volume, $V_{\mathrm{C}_{1}}$, as ice II containing no hydrogen and that the dependence $V_{\mathrm{C}_{1}}(P, T)$ in a hydrogen atmosphere is described by the equation of state for $\mathrm{D}_{2} \mathrm{O}$ ice II from ref 15. At pressures from 2.5 to $4.5 \mathrm{kbar}$ and temperatures from -50 to $+25^{\circ} \mathrm{C}$, without loss of accuracy, this equation can be approximated as

$$
V_{\mathrm{C}_{1}}=15.59-0.106 P+0.0045 T
$$

where $P$ is in kbar, $T$ in ${ }^{\circ} \mathrm{C}$, and $V_{\mathrm{C} 1}$ in $\mathrm{cm}^{3} / \mathrm{mol} \mathrm{H}_{2} \mathrm{O}$. The value of $V_{\mathrm{C}_{1}}$ at the $\mathrm{L}+$ sII $+\mathrm{C}_{1}$ triple point indicated in Table 2 and shown in Figure 5 by the solid star was calculated using this equation.

Hydrogen Contents of the $\mathbf{L}$ and sII Phases near the $\mathbf{L}+$ sII $+\mathbf{C}_{\mathbf{1}}$ Triple Point. To immediately obtain the hydrogen contents of phases from volumetric data, it is necessary to know the total amount of each component of the system in addition to the molar volume of each phase. The mass $M_{\mathrm{H}_{2} \mathrm{O}}$ of water in the condensed phases was always precisely known in our highpressure experiments, and it did not change with varying the temperature and pressure due to the very low water solubility in the hydrogen gas $^{9}$ in the investigated pressure range. At the same time, the total mass $M_{\mathrm{H}_{2}}$ of hydrogen in the system could only be estimated with an accuracy no better than $5 \%$ that resulted in an unacceptable error of $\delta X \approx 0.1$ in the hydrogen content of the condensed phases. We partly overcame this difficulty by determining the difference in the hydrogen contents of phases that were in equilibrium with each other and by evaluating the possible compositions of these phases from other considerations.

Using the condition that $M_{\mathrm{H}_{2}}$ does not change in the course of the sII $\rightarrow \mathrm{L}$ transition and substituting the volume $V_{\mathrm{L}}$ of the liquid phase from eq 1 , one gets the equation

$$
X_{\mathrm{L}}=\left(V_{\mathrm{L} 0}-V_{\mathrm{sII}}-\Delta V_{\mathrm{LsII}}+V_{\mathrm{H}_{2}} X_{\mathrm{sII}}\right) /\left(V_{\mathrm{H} 2}-\beta\right)
$$

At the $\mathrm{L}+\mathrm{sII}+\mathrm{C}_{1}$ triple point, at $3.6 \mathrm{kbar}$ and $1{ }^{\circ} \mathrm{C}$, eq 4 links the compositions of the sII and $\mathrm{L}$ phases in a unique fashion because all other parameters are known $\left(V_{\mathrm{L} 0}=15.99\right.$ $\mathrm{cm}^{3} / \mathrm{mol} ;{ }^{12} \Delta V_{\mathrm{L} \rightarrow \mathrm{sII}}$ is indicated in Table $1 ; V_{\text {sII }}$ and $V_{\mathrm{H}_{2}}$ in Table 2).

According to ref 6 , the hydrogen content of the sII phase reaches its maximum value of $X_{\text {sII }}=48 / 136 \approx 0.353$ at a hydrogen pressure of about $2 \mathrm{kbar}$ when the temperatures is lowered to $50 \mathrm{~K}$. If the sII phase has the same composition at

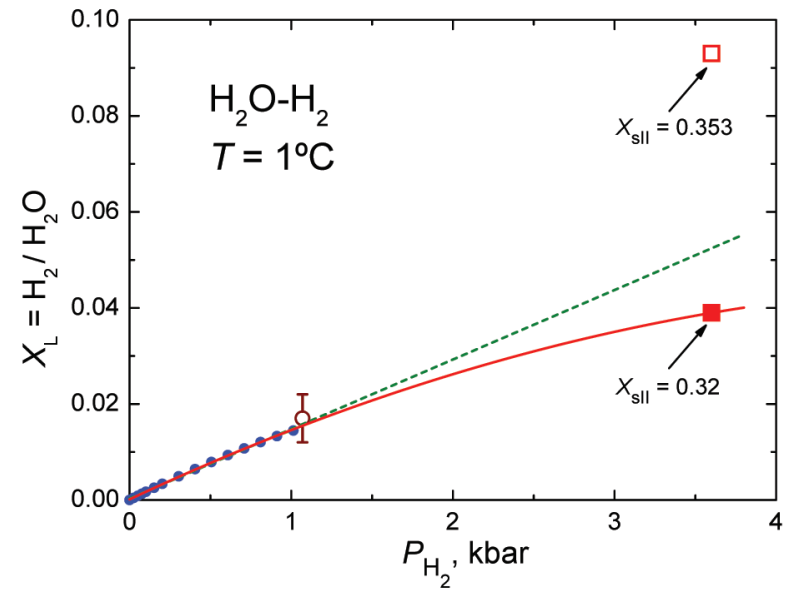

Figure 6. Hydrogen solubility in liquid water as a function of hydrogen pressure. The solid circles are the experimental data at $1{ }^{\circ} \mathrm{C}$. ${ }^{19}$ The dashed line is the linear fit to this data. The squares show the $\mathrm{H}$ content of the liquid at the $\mathrm{L}+\mathrm{sII}+\mathrm{C}_{1}$ triple point resulting from eq 4 with the $X_{\text {sII }}$ values indicated as labels. The open circle presents the composition of the liquid at the $\mathrm{L}+\mathrm{I}_{\mathrm{h}}+$ sII triple point. ${ }^{8}$

the $\mathrm{L}+\mathrm{sII}+\mathrm{C}_{1}$ triple point, then eq 4 yields $X_{\mathrm{L}}=0.093$ for the liquid at this point. As one can see from Figure 6, such a value of $X_{\mathrm{L}}$ is improbably large.

The solubility of hydrogen in water was earlier measured at temperatures $0-100{ }^{\circ} \mathrm{C}$ and hydrogen pressures up to 1000 atm. ${ }^{19}$ A linear extrapolation (dashed line in Figure 6) of the experimental isotherm at $1{ }^{\circ} \mathrm{C}$ gives $X_{\mathrm{L}}=0.052$ at $3.6 \mathrm{kbar}$. This is the upper limit for $X_{\mathrm{L}}$ at the triple point because the experimental isotherm (solid circles) is convex. In order that the $\mathrm{H}$ content of the liquid phase resulting from eq 4 comes down from $X_{\mathrm{L}}=0.093$ (open square in Figure 6) to a more feasible value of $X_{\mathrm{L}}=0.04$ (solid square), the conjugate sII phase should have $X_{\text {sII }}=0.32$. An extrapolation of the experimental $X(P)$ isotherms for the $\mathrm{H}_{2} \mathrm{O}-\mathrm{H}_{2}{ }^{5,7}$ and $\mathrm{D}_{2} \mathrm{O}-\mathrm{D}_{2}{ }^{8}$ systems to higher pressures demonstrates that $X_{\text {sII }}=0.32$ is a very likely composition of the sII phase at $3.6 \mathrm{kbar}$.

The isotherms in refs 5, 7, and 8 looked similar at all investigated temperatures, from -40 to $-10^{\circ} \mathrm{C}$. For illustration (Figure 7) we chose one of the $\mathrm{D}_{2} \mathrm{O}-\mathrm{D}_{2}$ isotherms ${ }^{8}$ because those had been measured more accurately than the $\mathrm{H}_{2} \mathrm{O}-\mathrm{H}_{2}$ isotherms. ${ }^{5,7}$ The portion of the isotherm at pressures above 1 kbar represents the dependence of the equilibrium hydrogen solubility in the sII phase. A linear extrapolation of this dependence to 3.6 kbar gives $X_{\text {sII }} \approx 0.35$, nearly coinciding with the maximum value of $X_{s I I}^{\max }=48 / 136$ that the sII phase can have. ${ }^{6}$ However, there are no reasons to think that $3.6 \mathrm{kbar}$ is a singular point of the $X_{\mathrm{sII}}(P)$ dependence, at which $X_{\mathrm{sII}}$ reaches the maximum value and then stops changing. It is much more likely that this dependence approaches $X_{s I I}^{\max }$ asymptotically.

As seen from Figure 7a, a smooth prolongation of the experimental $X_{\text {sII }}(P)$ dependence so as to asymptotically approach $X_{s I \mathrm{max}}^{\max }=48 / 136$ (horizontal dashed line) at increasing pressure requires that $0.32 \leq X_{\text {sII }}(3.6 \mathrm{kbar}) \leq 0.34$. This interval of the admissible $X_{\text {sII }}(3.6 \mathrm{kbar})$ values was the same for every $X_{\mathrm{SII}}(P)$ isotherm measured at temperatures from -40 to -10 ${ }^{\circ} \mathrm{C}^{5,7,8}$ and could therefore be extrapolated without change to a temperature of $+1{ }^{\circ} \mathrm{C}$ of the $\mathrm{L}+$ sII $+\mathrm{C}_{1}$ triple point. We adopted $X_{\text {sII }}(3.6 \mathrm{kbar})=0.32$ as the hydrogen content of the sII phase in equilibrium with the $\mathrm{L}$ phase at the triple point in order to get a plausible value of $X_{\mathrm{L}}=0.04$ from eq 4 .

It should be emphasized that the achieved consistency of the $X_{\text {sII }}$ and $X_{\mathrm{L}}$ values linked with eq 4 is only possible if $\Delta V_{\mathrm{L} \rightarrow \text { sII }}$ 


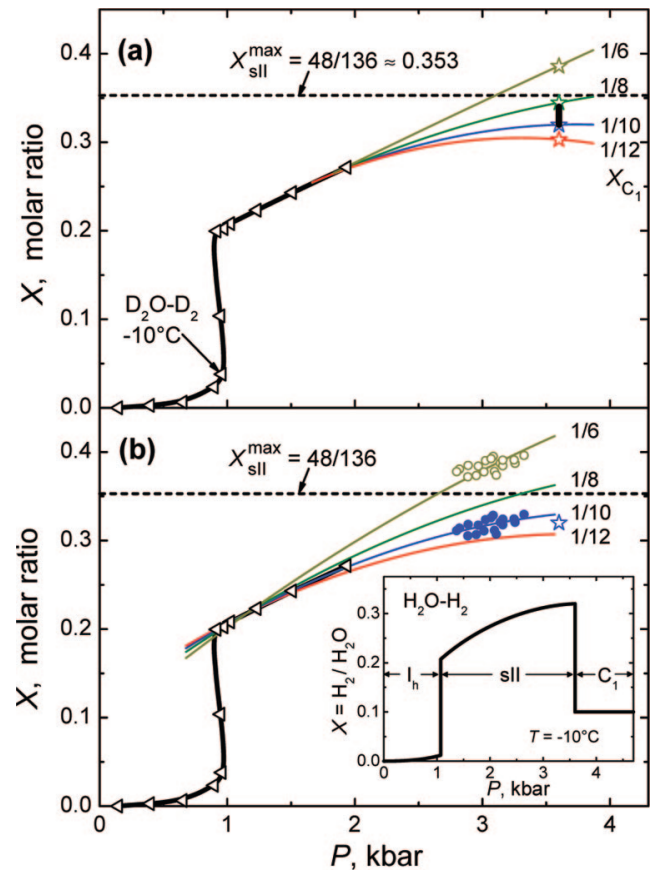

Figure 7. Isotherm of deuterium solubility in $\mathrm{D}_{2} \mathrm{O}$ ices at $-10{ }^{\circ} \mathrm{C}$ measured at decreasing pressure ${ }^{8}$ (open left triangles connected with the solid curve) and its extrapolation to higher pressures. The thick vertical segment in (a) shows the interval $0.32-0.34$ of the most likely $X_{\text {SII }}$ values at $3.6 \mathrm{kbar}$. The open stars in (a) and one star in (b) represent the $X_{\text {sII }}$ values at $3.6 \mathrm{kbar}$ and $1{ }^{\circ} \mathrm{C}$ resulting from eq 5 with the $X_{\mathrm{C}_{1}}$ values indicated on the right of the stars. The estimated $X_{\text {sII }}(P)$ dependences shown in (b) by the circles and thin solid curves are discussed in subsection "Hydrogen Content...". The inset shows an equilibrium isotherm of hydrogen solubility in $\mathrm{H}_{2} \mathrm{O}$ ices at $-10{ }^{\circ} \mathrm{C}$.

is equal to $0.35 \mathrm{~cm}^{3} / \mathrm{mol}$ as in Table 1 or has a larger positive value. For example, $\Delta V_{\mathrm{L} \rightarrow \text { sII }}=0$ would require $X_{\text {SII }}(3.6 \mathrm{kbar})$ $=0.30$ to get $X_{\mathrm{L}}=0.04$. As seen from Figure 7a, this $X_{\text {sII }}$ value is too low for the $X_{\text {sII }}(P)$ dependence to smoothly approach the $X_{\text {SII }}=48 / 136$ asymptote at increasing pressure. Since $\Delta V_{\mathrm{L} \rightarrow \text { SII }}$ $>0$ requires $(\mathrm{d} T / \mathrm{d} P)_{\mathrm{L} \leftrightarrow \mathrm{sII}}<0$ at the triple point, this justifies the occurrence of a maximum on the equilibrium melting curve of the sII phase (see Figure 2), in excellent agreement with DTA results of ref 2.

Hydrogen Content of the $\mathrm{C}_{1}$ Phase near the $\mathrm{L}+\mathrm{sII}+\mathrm{C}_{1}$ Triple Point. On the basis of results of Raman studies, the $\mathrm{C}_{1}$ phase was earlier assumed to be a stoichiometric compound with the maximum hydrogen content $X_{\mathrm{C}_{1}}=1 / 6 \approx 0.167$ possible from crystallographic considerations. ${ }^{3}$ Our estimates showed that the hydrogen content of the $\mathrm{C}_{1}$ phase at the $\mathrm{L}+\mathrm{sII}+\mathrm{C}_{1}$ triple point is smaller and its most likely value is $X_{\mathrm{C}_{1}}=0.1$.

The estimates were based on the equation

$$
X_{\mathrm{sII}}=X_{\mathrm{C}_{1}}+\left(V_{\mathrm{sII}}-V_{\mathrm{C}_{1}}+\Delta V_{\mathrm{sII} \rightarrow \mathrm{C}_{1}}\right) / V_{\mathrm{H}_{2}}
$$

resulting from the invariance of the total mass $M_{\mathrm{H}_{2}}$ of hydrogen in the course of the sII $\rightarrow \mathrm{C}_{1}$ transition. Using $\Delta V_{\mathrm{SII} \rightarrow \mathrm{C}_{1}}$ from Table $1, V_{\text {sII }}, V_{\mathrm{C}_{1}}$, and $V_{\mathrm{H}_{2}}$ from Table 2 , and $X_{\mathrm{C}_{1}}=1 / 6$ suggested in ref 3 , eq 5 yields $X_{\text {sII }}=0.39$ at the $\mathrm{L}+$ sII $+\mathrm{C}_{1}$ triple point. This significantly exceeds $X_{s I I}^{\max }=48 / 136 \approx 0.353$ assessed in ref 6.

There are two variables in eq $5, V_{\mathrm{C}_{1}}$ and $X_{\mathrm{C}_{1}}$, whose values are not known with certainty. If $X_{\mathrm{C}_{1}}=1 / 6$ is assumed to be correct, eq 5 will require $V_{\mathrm{C}_{1}}=15.90$ and $16.58 \mathrm{~cm}^{3} / \mathrm{mol}$ to get $X_{\text {SII }}=0.353$ and 0.32 , respectively, at $3.6 \mathrm{kbar}$ and $1{ }^{\circ} \mathrm{C}$. If the
$V_{\mathrm{C}_{1}}(P)$ dependence is further assumed to obey a BirchMurnaghan equation with the same $K_{0}$ and $K_{0}^{\prime}$ as ice II, ${ }^{15}$ an extrapolation to $21 \mathrm{kbar}$ and $22{ }^{\circ} \mathrm{C}$ will transform the two $V_{\mathrm{C}_{1}}$ values to those shown in Figure 5 with the half-blackened squares. As one can see from Figure 5, both points thus calculated are inconsistent with experiment (solid square), and the discrepancy is so large that it cannot be eliminated by changing the extrapolation method within reasonable limits.

This result evidences that $X_{\mathrm{C} 1}$ is significantly less than $1 / 6$ at the $\mathrm{L}+\mathrm{sII}+\mathrm{C}_{1}$ triple point. There is only one analogue of the $\mathrm{C}_{1}$ phase, the He-filled ice II. Its composition was earlier studied by neutron diffraction and shown to vary from $\mathrm{He} / \mathrm{D}_{2} \mathrm{O} \approx 1 / 12$ to $1 / 6$ depending on temperature and pressure, while the molar volume practically coincided with that of pure ice II. ${ }^{18} \mathrm{We}$ therefore examined the effect of varying the hydrogen content of the $C_{1}$ phase from $X_{C_{1}}=1 / 12$ to $1 / 6$ on the composition of the sII phase assuming that the $\mathrm{C}_{1}$ phase had the same molar volume as ice II.

The open stars in Figure 7 show results for the sII phase at the triple point calculated using eq 5 . As one can see, the compositions $1 / 10<X_{\mathrm{C}_{1}}<1 / 8$ of the $\mathrm{C}_{1}$ phase are most likely. The value of $X_{\mathrm{C}_{1}}=1 / 10$ corresponds to $X_{\mathrm{SII}}=0.32$ which gives an acceptable value of $X_{\mathrm{L}}=0.04$ if substituted in eq 4. The three compositions satisfy every constraint resulting from extrapolation of the known properties of $\mathrm{H}_{2} \mathrm{O}-\mathrm{H}_{2}$ phases to the vicinity of the $\mathrm{L}+$ sII $+\mathrm{C}_{1}$ triple point. These compositions are indicated in Table 2 together with the corresponding molar volumes.

As an additional test for self-consistency of the obtained results, we examined one more relation between the compositions of an $\mathrm{H}_{2} \mathrm{O}-\mathrm{H}_{2}$ sample in two single-phase states, sII and $\mathrm{C}_{1}$, at different hydrogen pressures:

$$
\begin{aligned}
X_{\text {sII }}^{\mathrm{s}}=X_{\mathrm{C}_{1}}^{\mathrm{c}}-\frac{V_{\mathrm{sys}}^{\mathrm{s}}-V_{\mathrm{sys}}^{\mathrm{c}}}{V_{\mathrm{H}_{2}}^{\mathrm{s}}} \frac{\mu_{\mathrm{H}_{2} \mathrm{O}}}{M_{\mathrm{H}_{2} \mathrm{O}}}+ \\
\left(1-\frac{V_{\mathrm{H}_{2}}^{\mathrm{c}}}{V_{\mathrm{H}_{2}}^{\mathrm{s}}}\right) \frac{M_{\mathrm{H}_{2}}^{\mathrm{c}}}{M_{\mathrm{H}_{2} \mathrm{O}}} \frac{\mu_{\mathrm{H}_{2} \mathrm{O}}}{\mu_{\mathrm{H}_{2}}}+\frac{V_{\text {sII }}^{\mathrm{s}}-V_{\mathrm{C}_{1}}^{\mathrm{c}}}{V_{\mathrm{H}_{2}}^{\mathrm{s}}}
\end{aligned}
$$

In this equation, variables with the superscripts "c" and "s" refer respectively to the point $\left(P_{\mathrm{H}_{2}}^{\mathrm{c}}, T\right)$ where the $\mathrm{C}_{1}$ phase is stable and to the point $\left(P_{\mathrm{H}_{2}}^{\mathrm{s}}, T\right)$ with the stable sII phase; $V_{\mathrm{sys}}^{\mathrm{s}}-V_{\text {sys }}^{\mathrm{c}}$ is the experimental difference between the two volumes of the $\mathrm{H}_{2} \mathrm{O}-\mathrm{H}_{2}$ system; $M_{\mathrm{H}_{2} \mathrm{O}}$ is the water mass; $M_{\mathrm{H}_{2}}^{\mathrm{c}}$ is the mass of the $\mathrm{H}_{2}$ gas at the $\left(P_{\mathrm{H}_{2}}^{\mathrm{c}}, T\right)$ point; $\mu_{\mathrm{H}_{2} \mathrm{O}}=18.015$ and $\mu_{\mathrm{H}_{2}}=2.016$ are the molecular masses of $\mathrm{H}_{2} \mathrm{O}$ and $\mathrm{H}_{2}$.

To make use of eq 6 , we proceeded on the assumptions that while constructing the isotherms of the hydrogen solubility in ices: (i) each of our $\mathrm{H}_{2} \mathrm{O}-\mathrm{H}_{2}$ samples completely transformed to the $\mathrm{C}_{1}$ phase when the pressure was increased to the maximum value; (ii) the hydrogen content of the $C_{1}$ phase did not change on further decrease in pressure until the $\mathrm{C}_{1} \rightarrow$ sII transition began; (iii) the sample completely transformed to the sII phase immediately after the $\mathrm{C}_{1} \rightarrow$ sII transition. Pressure dependences of the molar volumes of the sII and $\mathrm{C}_{1}$ phase were approximated by eq 2 and eq 3 , respectively.

The mass $M_{\mathrm{H}_{2}}^{\mathrm{c}}$ of the $\mathrm{H}_{2}$ gas in equilibrium with the $\mathrm{C}_{1}$ phase was determined by a least-squares fit of the equation of state of molecular hydrogen ${ }^{11}$ to the difference $\left(V_{\text {sys }}^{\mathrm{c}}-V_{\text {sys }}^{\mathrm{c} 0}\right)-\left(V_{\mathrm{C}_{1}}^{\mathrm{c}_{1}}-\right.$ $\left.V_{\mathrm{C}_{1}}^{\mathrm{co}}\right) M_{\mathrm{H}_{2} \mathrm{O}} / \mu_{\mathrm{H}_{2} \mathrm{O}}$ for the experimental points of the $\mathrm{C}_{1}$ phase on each $V(P)$ isotherm measured at decreasing pressure. One of the points located at $\left(P_{\mathrm{H}_{2}}^{\mathrm{co}}, T\right)$ was arbitrary taken as the computing 
origin. The typical quality of the fits is illustrated by the solid left triangles in Figure 3b.

Substituting the obtained $M_{\mathrm{H}_{2}}^{\mathrm{c}}$ value together with the values of $V_{\mathrm{sys}}^{\mathrm{c}}, V_{\mathrm{H}_{2}}^{\mathrm{c}}$, and $V_{\mathrm{C}_{1}}^{\mathrm{c}}$ for one of the fitting points in eq 6 gives a unique correspondence between the hydrogen content of the $\mathrm{C}_{1}$ phase and the hydrogen content of the sII phase at every point of the $V(P)$ isotherm below the pressure of the $\mathrm{C}_{1} \rightarrow$ sII transition. Using eq 6 , the $X_{\text {sII }}(P)$ dependences were calculated for all $V(P)$ isotherms and, within the experimental scatter, proved to be independent of the measuring temperature. The $X_{\mathrm{SII}}(P)$ points calculated for two compositions of $X_{\mathrm{C}_{1}}=1 / 6$ and $1 / 10$ at all temperatures are presented by two clusters of circles in Figure $7 \mathrm{~b}$. The thin solid curves in this figure show polynomial fits to the experimental $X_{\text {sII }}(P)$ dependence (open left triangles) and clusters of the $X_{\text {sII }}(P)$ points calculated for different values of $X_{\mathrm{C}_{1}}$. As one can see, the fitting line for $X_{\mathrm{C}_{1}}$ $=1 / 10$ is close to the point $X_{\text {SII }}(3.6 \mathrm{kbar})=0.32$ shown by the open star and calculated for $X_{\mathrm{C}_{1}}=1 / 10$ more accurately using eq 5. Assuming that the $X_{\text {sII }}(P)$ dependence should asymptotically approach $X_{s I I}^{\max }=48 / 136$ at increasing pressure, the behavior of the fitting lines confirms that the hydrogen content of $X_{\mathrm{C}_{1}} \approx 0.1$ is most likely for the $\mathrm{C}_{1}$ phase.

$X_{\mathrm{C}_{1}}=0.1$ corresponds to a concentration of $9.1 \mathrm{~mol} \% \mathrm{H}_{2}$ in the $\mathrm{C}_{1}$ phase that is below the bottom limit of the interval 10-20 mol $\% \mathrm{H}_{2}$ established for the $\mathrm{C}_{1}$ phase in ref 3 . Nevertheless, we are not inclined to consider this as a serious discrepancy because the estimate of ref 3 was based on the intensities of peaks in the Raman spectra, and such a method can overrate the hydrogen concentration in hydrates. For example, the preliminary estimate of the hydrogen content of the sII phase using this method ${ }^{4}$ proved later to be overrated by $33 \% .^{6}$

The inset in Figure $7 \mathrm{~b}$ shows the behavior of the equilibrium hydrogen solubility in ices that should be observed at temperatures below the $\mathrm{L}+\mathrm{I}_{\mathrm{h}}+$ sII triple point according to results of refs 5 and 8 and the present work. An interesting effect is the decrease in the hydrogen content of ice after the sII $\rightarrow \mathrm{C}_{1}$ phase transition. In most systems with gaseous hydrogen, an increase in the $\mathrm{H}_{2}$ pressure leads to the increase in the hydrogen solubility in the condensed phases. The reverse effect accompanying the sII $\rightarrow \mathrm{C}_{1}$ transition is a consequence of the looseness of clathrate structure of the sII phase. The molar volume of this phase is by $2.01 \mathrm{~cm}^{3} / \mathrm{mol}$ higher than the total volume of the $C_{1}$ phase and gaseous hydrogen liberated from the sII phase in the course of the transition (see Table 1). The negative change in volume, $\Delta V$, gives rise to the thermodynamic driving force $\int \Delta V \mathrm{~d} P$ causing the phase transition at increasing pressure in accordance with Le Chatelier's principle.

\section{Conclusions}

With the line of the sII $\leftrightarrow \mathrm{C}_{1}$ transformation constructed in the present work, the limits of the stability region of the sII phase are fully identified (Figure 2). The melting lines of the sII and $\mathrm{C}_{1}$ phases determined by DTA in ref 2 are re-examined and shown to accurately represent the phase equilibria near the $\mathrm{L}+\mathrm{sII}+\mathrm{C}_{1}$ triple point. In particular, the negative slope of the sII $\leftrightarrow \mathrm{L}$ curve near the triple point and therefore the occurrence of a maximum on this curve at a lower pressure proved to be a requisite for the consistency of hydrogen contents of the sII and L phases. To our knowledge, among the $T-P$ diagrams of a large body of gas-water systems studied so far, the diagram of the $\mathrm{H}_{2} \mathrm{O}-\mathrm{H}_{2}$ system is the first, in which equilibrium lines of phase transformations between solid phases have been constructed.

The obtained volumetric data also allowed a reliable determination of the volume and entropy effects of phase transitions near the $\mathrm{L}+\mathrm{sII}+\mathrm{C}_{1}$ triple point (Table 1 ). The analysis of the available literature data gave plausible values of the molar volumes of the sII and $\mathrm{L}$ phases and a reasonable estimate of the volume of the $\mathrm{C}_{1}$ phase (Table 2). Combining these values of the molar volumes with results of the volumetric measurements, the hydrogen content of each condensed phase at the triple point was estimated (Table 2).

Acknowledgment. This work was supported by the Russian Academy of Sciences (the program "Physics and Mechanics of Strongly Compressed Matter"), the Russian Foundation for Basic Research (projects nos. 05-02-17733 and 08-02-00846), and the Foundation for Support of Russian Science.

\section{References and Notes}

(1) Tonkov, E. Yu. High Pressure Phase Transformations: A Handbook; Gordon \& Breach: Philadelphia, 1992; pp 448-449.

(2) (a) Dyadin, Yu. A.; Larionovnt, E. G.; Manakov, A. Yu. Zh. Strukt. Khim. 1999, 40, 974-980. (b) Dyadin, Yu. A.; Larionov, E. G.; Manakov, A. Yu.; Zhurko, F. V.; Aladko, E. Ya.; Mikina, T. V.; Komarov, V. Yu. Mendeleev Commun. 1999, 209-210.

(3) Vos, W. L.; Finger, L. W.; Hemley, R. J.; Mao, H.-K. Phys. Rev. Lett. 1993, 71, 3150-3153.

(4) Mao, W. L.; Mao, H.-K.; Goncharov, A. F.; Struzhkin, V. V.; Guo, Q.; Hu, J.; Shu, J.; Hemley, R. J.; Somayazulu, M.; Zhao, Y. Science 2002, 297, 2247-2249.

(5) Efimchenko, V. S.; Antonov, V. E.; Barkalov, O. I.; Beskrovnyy, A. I.; Fedotov, V. K.; Klyamkin, S. N. High Pressure Res. 2006, 26, 439443.

(6) Lokshin, K. A.; Zhao, Y.; He, D.; Mao, W. L.; Mao, H.-K.; Hemley, R. J.; Lobanov, M. V.; Greenblatt, M. Phys. Rev. Lett. 2004, 93, 125503$1-125503-4$.

(7) Barkalov, O. I.; Klyamkin, S. N.; Antonov, V. E.; Efimchenko, V. S. JETP Lett. 2005, 82, 413-415.

(8) Efimchenko, V. S.; Antonov, V. E.; Barkalov, O. I.; Klyamkin, S. N. J. Phys. Chem. B 2008, 112, 7026-7031.

(9) Mao, W. L.; Mao, H.-K. Proc. Natl. Acad. Sci. U.S.A. 2004, 101, 708-710.

(10) Baranowski, B.; Tkacz, M.; Bujnowski, W. Roczniki Chem. 1975, $49,437-439$. 92.

(11) Tkacz, M.; Litwiniuk, A. J. Alloys Compd. 2002, 330-332, 89-

(12) Bridgman, W. B. J. Chem. Phys. 1935, 3, 597-605.

(13) Tiepel, E. W.; Gubbins, K. E. J. Phys. Chem. 1972, 76, 30443049.

(14) Moore, J. C.; Battino, R.; Rettich, T. R.; Handa, Y. P.; Wilhelm, E. J. Chem. Eng. Data 1982, 27, 22-24.

(15) Fortes, A. D.; Wood, I. G.; Alfredsson, M.; Vočadlo, L.; Knight, K. S. J. Appl. Crystallogr. 2005, 38, 612-618.

(16) Gagnon, R. E.; Kiefte, H.; Clouter, M. J. J. Chem. Phys. 1990, 92, 1909-1914.

(17) Londono, D.; Kuhs, W. F.; Finney, J. L. Nature (London) 1988, $332,141-142$.

(18) Londono, D.; Finney, J. L.; Kuhs, W. F. J. Chem. Phys. 1992, 97, $547-552$.

(19) Wiebe, R.; Gaddy, V. L. J. Am. Chem. Soc. 1934, 56, 76-79.

\section{JP807996H}

